\title{
Voice Controlled Data Acquisition Car Based on Zigbee Technology
}

\author{
Dinu Mathew \\ ME, Electronics and TeleCommunication, Sathyabama University, Chennai,India
}

\begin{abstract}
The objective of the research is to design a voice controlled car and use it as a platform for obtaining feedback from a critically controlled environment. The system comprises of a user interface module and a Robocar. The user interface includes an electret microphone through which the voice commands will be accepted by the voice recognitionchip(HM2007).Microcontrollers(PIC16F877A) in the transmitting and receiving side controls the motion of Robocar. The control commands and data are transmitted and received using ZigBee (IEEE 802.15.4).Sensors and transducers are present in the Robocar for collecting application specific datas.
\end{abstract}

Keywords:Voice controlled,Zigbee, User interface, Real time.

\section{INTRODUCTION}

Human interaction with robots and electronic gadgets using speech is used widely than other communication techniques. The system generally consists of voice response and speech recognition subsystems. The Speech interactive system is a complete easy to build programmable speech recognition and synthesis circuit. Speech recognition allows you to provide input to an application with your voice. In the desktop world you need a microphone to be able to do this. In the near future speech recognition will become the method of choice for controlling appliances,toys,tools,computers and robotics. It can also be used in defence applications. This research details the construction and building of a stand alone trainable speech recognition circuit that may be interfaced to control the Robocar and the IP camera mounted on the Robocar.The circuit is trained to recognise words that you want it to recognise. To control and command the Robocar by speaking to it will make it easier while increasing the efficiency and effectiveness of working with it.

Currently most speech recognition systems available today are programs that use personal computers. The add-on programs operate continuously in the background of the computers,operating system. The disadvantage in this approach is the necessity of a computer, portability and security. In this research we use a voice recognition chip instead of computer to process the input analog voice signals.

The speech recognition subsystem was built using Hualon Microelectronics corporation's HM2007 speech recognition chip. The microcontroller used for generating the control commands is PIC16F877A.It is manufactured by Microchip Technologies. The motion of the IP camera can be controlled by the user. Image acquisition at multiple angles can be obtained by the movement of the IP camera.

\section{SYSTEM DESCRIPTION}

A. $\quad$ Literature review.

There are many scientists and researchers who develop computer software that can recognize human voice commands in so many languages such as English,Japanese and Thai.There are many techniques that are used to recognize voice commands which are:1)Neural Network Method 2)Fuzzy logic technique3)hidden Markov model and 4)Gaussian mixture model etc[1][2].

Researchers transform sound wave into digital wave by a computer. After that they use digital signal to manage different electronic equipments, for example 1)controlling robot arm movement 2)helping the handicapped to move a wheel chair etc.

The idea of this research came from a paper presented in IEEE in the year 2010.The paper presented was on the "Operation of a radio controlled car by voice commands". This paper presented has its own limitations. The proposed car implemented by us is expected to overcome these limitations and provide more area for development with this as the platform.

First of all let us give a brief idea of the paper presented in IEEE and later we will deal with how our proposed Robocar is superior to the above. The objective of this work was to operate a radio controlled car by using the voice commands. Here the user voice commands were given to a computer machine and converted it into digital signal. After that the digital signal was converted into a radio wave commands. The RC car operated according to these radio wave commands[2][3][11][12]. 
Following were the major limitations of this RC car:-

- Efficiency low in noisy environment.

- Complexity increased due to the use of a computer system.

- Requires continuous AC supply.

- Range and direction is limited.

B. Advantages of the proposed robocar.

- Voice commands are processed by the voice recognition chip and the microcontroller.

- Use of ZigBee eliminates noise interference and provides high security.

- Long range transmission and reception is possible.

- Robocar can be moved in any direction as per the user commands.

- Application specific datas can be accurately collected.

\section{SYSTEM DESIGN}

This part introduces our approach of creating a system of an Robo Car which is controlled by the voice commands of the user. We will start with the overall frame work of the system and the description of each component in the framework and the basic understanding of the technique we are using in each component. The system consists of a Control Module and a RoboCar.

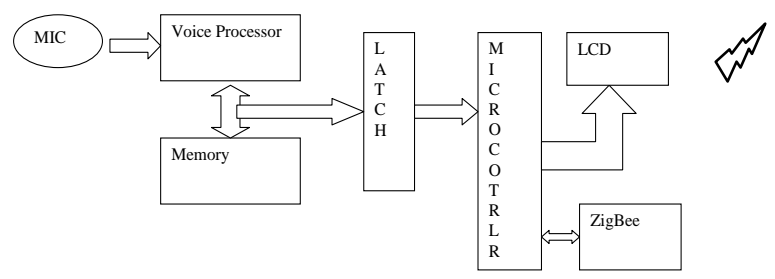

Figure 1. The system block diagram of the Control Module

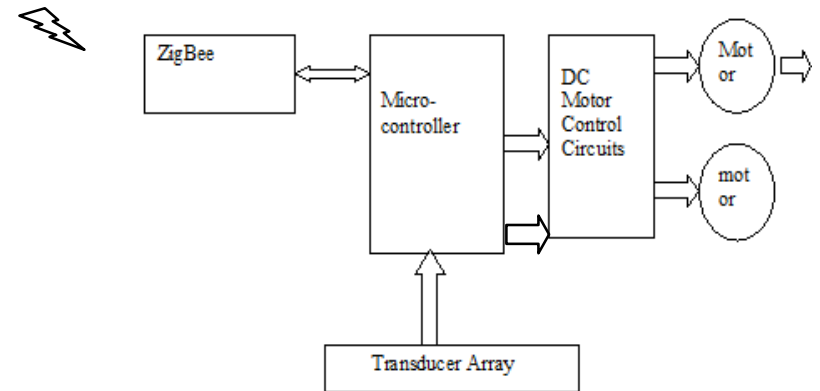

Figure 2. The system block diagram of the Robo Car

\section{A. $\quad$ Working Principle}

The system comprises of a user interface module and a robocar. The user interface includes an electret microphone through which the voice commands will be accepted by the voice recognition chip(HM2007) in the training mode. These voice signals are converted into an 8 bit pattern using delta sampling and these patterns are mapped to an 8 bit integer code. The integer code can be selected by using a $4 \times 3$ matrix keyboard which is also interfaced to the control module. Each pattern will be stored in an 8x8K external memory(CY6264).

After the training mode the system comes into the recognition mode. In this mode when a voice command input through the microphone will be again converted into an 8 bit pattern and will be compared with the stored pattern. If any match between the patterns are found then the voice processing chip will place the corresponding 8 bit integer code into its data bus. Data bus contents are then latched to an 8 bit Microcontroller (PIC16F877A) and the code data accepted by the controller is processed and the required commands will be send through a ZigBee which is controlled by the USART; a built in peripheral facility available with the Microcontroller for serial data transmission. An LCD display(LM016L) will also be there to verify the integer code. The control commands transmitted through the ZigBee is received by another ZigBee in the receiver side and these signals are given to another Microcontroller. The received codes are decoded and appropriate signals are generated to control the motor of the Robocar. In the Robocar there are some sensors and transducer arrays which picks up the required parameters of the environment and processing circuits are there to refine these signals. These signals are transmitted back to the control module using ZigBee[5][10]. The received parameters are displayed in the LCD display. 


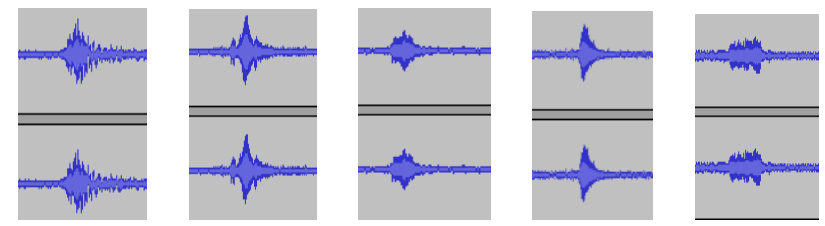

Figure 3. The sound wave frequencies of five voice commands which are :a)Left b)Reverse c)Right d)Start e)Stop

\section{SYSTEM OVERVIEW}

The entire system is portable and battery operated. The use of Microcontroller having built in peripheral facilities like I/O PORTS,USART etc reduces circuit complexity very much as well as the cost. Communication between the user interface and the robocar is done using ZigBee. The modulation technique used in ZigBee is Spread Spectrum modulation. High noise immunity is one of the major characteristics of Spread Spectrum modulation.

An important attribute of Spread Spectrum modulation is that it provides protection against externally generated interfering signals with finite power. The Robocar platform can be used for various applications like data acquisition, image collection, spywork etc by interfacing appropriate transducers at the Robocar. Control of the Robocar using keyboard is also possible which increases the system reliability and efficiency when silent operation is preferred.

A. Platform

- Embedded Technology is used to develop the project.

- Microcontroller used is PIC16F877A is the heart of the system.

- Signal processing is accomplished with HM2007 chip, voice recognition chip.

- ZigBee technology based on IEEE 802.15.4 protocol is implemented for wireless communication.

- 8 x8K SRAM is used to store the pattern.

B. HM2007(Voice Recognition Chip)

HM2007 is a single chip CMOS voice recognition LSI circuit with on chip analog front end, voice analysis, recognition process and system control functions. A 40 isolated -word voice recognition system can be composed of external microphone, keyboard, 8x8KSRAM and some other components. Combined with the microcontroller an intelligent recognition system can be built.

To communicate with the 8x8KSRAM, there is a 13-bit address bus and a 8-bit data bus as well as memory read/write pin and a memory enable pin. To communicate with the keypad , there is a 4-bit wide K-bus used for passing data to the HM2007, and a 3-bit S-bus, used for sending commands to the HM2007[7][8][11][12].

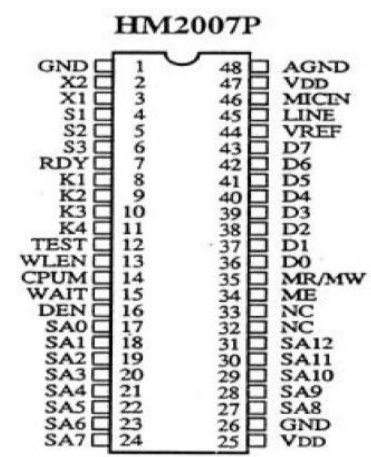

Figure 2. Voice processing Chip

Features:

- Speaker dependent isolates voice recognition system.

- Maximum 40 words can be recognized for one chip.

- Maximum 1.92sec of word can be recognized.

- Multiple chip configuration is possible.

- Two control mode is supported:Manual mode and CPU mode.

- Response time is less than $300 \mathrm{~ms}$.

- 48-pin DIP,52 pin PLCC,48 pad bare chip. 


\section{ZigBee Wireless Technology}

A type of low cost, low power wireless technology which is used for the different purposes at ultra low power is known as ZigBee Technology. It was not proposed or designed for the high speed data transfer applications. But it was designed for working on excellent long battery timings at low cost and also at ultra low power consumption.

The IEEE 802.15.4 standard is a simple packet data protocol for light weight wireless networks and specifies the Physical (PHY) and Medium Access Control (MAC)layers for Multiple Radio Frequency bands, including $868 \mathrm{MHz}, 915 \mathrm{MHz}$ and $2.4 \mathrm{GHz}$. The IEEE 802.15 .4 standard is designed to provide reliable data transmission of modest amount of data upto 100 meters or more while consuming very little power. Zigbee utilizes Spread Spectrum modulation technique for communication [4][5][10].

ZigBee technology takes full advantage of the IEEE 802.15.4 standards and extend the capabilities of this new radio standard by defining a flexible and secure network layer that supports a variety of architectures to provide high reliable wireless communication. High throughput and low latency for low duty cycle applications that are nearly to $0.1 \%$.ZigBee-PRO can provide a range of upto $1.5 \mathrm{Km}$.

\section{Flow Control}

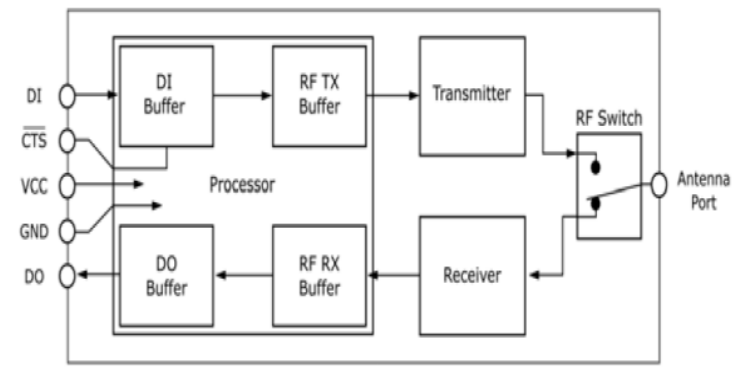

Figure 3. Internal Data Flow Diagram

- $\quad$ DI (Data In) Buffer:

When serial data enters the RF module through the DI pin (pin 3), the data is stored in the DI Buffer until it can be processed. When the DI buffer is 17 bytes away from being full; by default, the module de-asserts CTS (high) to signal to the host device to stop sending data. CTS is re-asserted after the DI Buffer has 34 bytes of memory available.

- $\quad$ DO (Data Out) Buffer:

When RF data is received, the data enters the DO buffer and is sent out the serial port to a host device. Once the DO Buffer reaches capacity, any additional incoming RF data is lost. If RTS is enabled for flow control, data will not be sent out the DO Buffer as long as RTS is de-asserted.
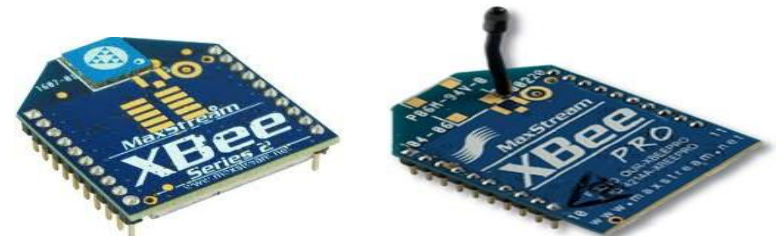

Figure 4. ZigBee and ZigBee-PRO

\section{PIC16F877A(Microcontrollor)}

The PIC16F877A is one of the most popular PIC microcontrollers and it comes in a 40pin DIP pin out and it has many internal peripherals. The 40 pins makes it easier to the peripherals as the functions are spread out over the pins. One of the main advantage is that each pin is only shared between two or three functions so its easier to decide what the pin function is.

Peripheral Interface Controller(PIC)Microcontroller is the platform on which we have developed our work.PIC 16F877A(Microchip Technology Inc) is an 8bit microcontroller, with a 25Mhz processor,33 input/output pins, $\left(8 \mathrm{~K}^{*} 14\right.$ words)of Enhanced FLASH program memory, (368*8bytes)of RAM,(256*8bytes)of data EEPROM. The PIC does not have an operating system and simply runs the program in its memory when it is turned on. Ease of design and Programmability and less power consumption are the main features of this microcontroller.

A disadvantage of the device is that it has no internal oscillator so you will need an external crystal of other clock source[11][12[13][14]. 


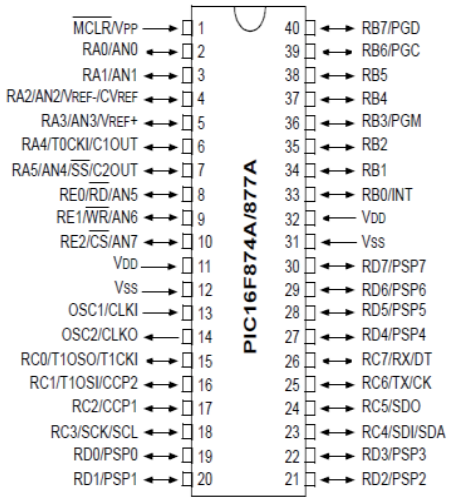

Figure 5. Microcontroller used is PIC16F877A

\section{SYSTEM IMPLEMENTATION}

This section explains the system requirements for both hardware and software implementation, which have the following details.

\section{A. Hardware Implementation}

The Hardware part of the system mainly consists of a Control Module and a RoboCar Circuit.

- ThehardwarepartofthecontrolmoduleincludesVoiceProcessingchip(HM2007),64KSRAM(CY6264)Microco ntroller(PIC16F877A),Latch(74HC573).

- The hardware part of the RoboCar circuit includes the Microcontroller,DC motors,LM35(Temperature Sensors),Motor Drivers(L293D).

- A 4x3 keyboard is used for generating the input code.

- Glass Epoxy PCB and General PCB is used.

- DC motors are used for the operation of the wheels.

B. Software Implementation

- PIC C Compiler is used for Programming the Microcontrollers.

- Embedded $\mathrm{C}$ is the Programming language used .

- Proteus 7 is used for simulation of the circuit .

- ARES is the software used for designing the PCB layout.

- WINPIC 800 is the software used for burning the program into the IC.

\section{EXPERIMENTAL RESULTS}

This part presents the experimental result of the RoboCar controlled by the voice commands given by the user which is developed and based on the concepts designed and mentioned in the previous part.In this system the experimental results mainly focus on the usability and effectiveness of the system.

\section{A. Usability Proof}

In this section we will analyse the usability of the operation of an RoboCar by the voice commands.The voice commands given by a single user are processed using a voice processor chip and convert them into digital signal. Microcontrollers generate the corresponding control code and transmit it using ZigBee and the Robocar moves accordingly.

The required parameters(temperature) are collected and send back to the user and is displayed on the LCD display. ZigBee provides high noise immunity due to Spread Spectrum modulation . The usability concept is proved by observing that our Robocar operate more efficiently than an RC Car controlled by a remote control.

\section{B. Effectiveness Proof}

The effectiveness test is done in the same way as in the previous section, but we focus on the correctness of the final result. We have tested under three different environments which are: 1) a quiet room 2) an office room and 3) a noisy room. We test each commands many times under each environment and the results of effectiveness-testing are obtained. The precision rates of the experiments are $99.0 \%$ and $94.20 \%$ in the quiet room and noisy room respectively. 
Voice Controlled Data Acquisition Car Based on Zigbee Technology

TABLE I. EFFECTIVENESS TESTING RESULT

\begin{tabular}{|c|c|c|c|c|c|}
\hline \multirow[t]{2}{*}{ Command } & \multirow[t]{2}{*}{$\begin{array}{c}\text { No of } \\
\text { Testings }\end{array}$} & \multicolumn{2}{|c|}{ Quiet Room } & \multicolumn{2}{|c|}{ Noisy Room } \\
\hline & & Right & Wrong & Right & Wrong \\
\hline Left & 100 & $100 / 100$ & $0 / 100$ & $95 / 100$ & $5 / 100$ \\
\hline Reverse & 100 & $99 / 100$ & $1 / 100$ & $94 / 100$ & $6 / 100$ \\
\hline Right & 100 & $99 / 100$ & $1 / 100$ & $93 / 100$ & $7 / 100$ \\
\hline Start & 100 & $98 / 100$ & $2 / 100$ & $94 / 100$ & $6 / 100$ \\
\hline Stop & 100 & $99 / 100$ & $1 / 100$ & $95 / 100$ & $5 / 100$ \\
\hline Total & 500 & $495 / 500$ & $5 / 500$ & $471 / 500$ & $29 / 500$ \\
\hline Precision & & $99 \%$ & $1.00 \%$ & $94.20 \%$ & $5.80 \%$ \\
\hline
\end{tabular}

VII. CONCLUSION

Based on the experiments results in the previous section we have fulfilled our research objective. We can conclude that the Robocar moves accurately as per the user voice commands in all different environmental conditions and is able to retrieve the specified data from the remote environment.

High security can be obtained since it is operated by a single user. Complexity of the system is highly reduced and efficient compared to the RC car discussed above.

The only disadvantage of this system is that the Robocar may misinterpret words having same frequencies and operate in the wrong way. Words have to be said in such a way that they are of different frequencies.

\section{References}

[1] Xiaoling Lv, Minglu Zhang and Hui Li, "Robot Control based on Voice Command", The Proceeding of the IEEE International Conference on Automation and Logistics, China 2008.

[2] Chomtip Pornpanomchai, Thammarat Saengsopee and Teravit Wongseree, "Robot Arm Control by Using Thai Voice Commands", The $1^{\text {st }}$ Northeastern Computer Science and Engineering Conference, Thailand, 2005.

[3] Parichart Leechor, Chomtip Pornpanomchai, "Operation of a Radio-Controlled Car by Voice Commands", $2^{\text {nd }}$ International Conference on Mechanical and Electronics Engineering, Thailand, 2010.

[4] Zigbee/IEEE 802.15.4 Networking Examples, Zigbee Wireless Network and Transceivers, 2008.

[5] Paolo Baronti, Prasant Pillai.Wireless Sensor Networks:A survey on the state of the art and the 802.15.4 and ZigBee Standards,Computer Communications, Volume 30,Issue 7,26 May 2007

[6] N.Yamasaki and Y.Anzai,"Active Interface for Human Robot Interaction,"Proccedings of the 1995 International Conference on Robotics and Automation,Nagoyo,Japan,May 1995.

[7] HM2007 Manual,Images Company

[8] Holmes J and Holmes W 2001 Speech Recognition and Synthesis 1 st Edition pp2,ISBN-10.

[9] Nedevshi S,Patra R A and Brewer E A 2005 Hardware Speech Recognition for User Interfaces in Low Cost ,Low Power Devices

[10] Li Wenfeng,Hanfei,"Short Distance Wireless Voice Communication Based on ZigBee"University Of Science and Technology,China,2007

[11] Mohammed Fezari and Mounir Bousbia-Salah,"A Voice Command system for Autonomous Robot Guidance", The $9^{\text {th }}$ IEEE International Workshop on Advanced Motion Control,pp.261-265,Istanbul,Turkey,2006.

[12] Peter X.Liu, A.D.C.Chen, R.Chen, K.Wang and Y.Zhu, "Voice Based Robot Control", The Proceeding of the 2005 IEEE International Conference on Information Acquisition,pp.543-547HongKong and Macau,China,2005.

[13] Martin Urban and Peter Bajscy, "Fusion of Voice, Gesture and Human-Computer Interface Control for remotely Operated Robot", The International Conference on Information Fusion,"pp.1644-1651,2005.

[14] D.S.Lees and L.J Leifer, "A Graphical Programming Language for Robots Operating in Lightly Structured Environments", Proceedings of the 1993 IEEE International Conference on Robotics and Automation, San Diego,California,May,pp.648-653,1993. 